\title{
An evaluative study to find the effectiveness of two methods of management of pediculosiscapitis among children of selected schools of Udupi District
}

\author{
${ }^{1}$ Ms. Manjula, ${ }^{2}$ Dr. Suja Karkada, ${ }^{3}$ Ms. Ansuya, ${ }^{4}$ Dr. Asha Kamath, ${ }^{5}$ Dr. Suneel \\ Mundkur \\ ${ }^{I}$ Asst. Professor, Dept of Community Health Nursing, Manipal College of Nursing, Manipal University, Manipal \\ ${ }^{2}$ Asst. Professor, RAK Medical and Health Science, RAK University, U.A.E. \\ ${ }^{3}$ Asst. Professor, Dept of Community Health Nursing, Manipal College of Nursing, Manipal University, Manipal \\ ${ }^{4}$ Senior Grade Lecturer, Dept of Community Medicine, Manipal University, Manipal. \\ ${ }^{5}$ Associate Professor, Dept of Pediatrics, Kasturba Hospital, Manipal
}

\section{Introduction}

Pediculosis is an important communicable infestation posing a public health problem in India. Infestation with pediculushumanuscapitis (head louse ) is more commonly observed among population who live in an overcrowded and insanitary environment. Though pediculosis can occur in any age group, younger children are more frequently affected as they probably have more contact with other affected children in overcrowded houses and schools. This infestation adversely affects not only their health but also their curricular activities. ${ }^{1}$

Currently, pediculosis affects 6-12 million persons in the United States each year, and this number continues to rise. Social stigma and persistent misconceptions complicate the implementation of appropriate management strategies. Diagnosis is made on the basis of finding nits (i.e., silvery-white eggs firmly attached to the hair shaft), concentrated on the crown, behind the ears, and at the nape of the neck. Transmission occurs by direct contact with an infested person or indirectly by contact with clothing, personal grooming articles, bedding, or upholstered furniture containing viable nits or lice. Although three chemical agents are currently available; permethrin 1.0\% (Nix Crème Rinse) is the treatment of choice. Environmental treatment is also necessary for the eradication of the infestation. Health care personnel who come in contact with this population need to be well informed of the facts in order to disseminate accurate information for diagnosis and management. $^{2}$

Indian studies have reported a wide - ranging prevalence of pediculosis in different groups, $51.26 \%$ among villagers, $6.4 \%$ among school children, and $4.6 \%$ in the semi urban community. Pediculosis is one of the most common parasitic infestations in the community, responsible for a high level of morbidity. The condition in most cases simply reflects poor hygiene. A house to house survey was conducted in 5 villages of Wardha district, Maharastra,with a combined population of 2063. A study conducted among 337 males and 329 females up to age 14 years found a prevalence rate of pediculosiscapitis infestation of $20.42 \%$. Infestation was detected in $8.9 \%$ of males and $32.2 \%$ of females. The infestation rate increased from $9.31 \%$ to $30.17 \%$ with the age group from $0-4$ years to $10-14$ years. $54.4 \%$ of children did not wash their hair daily; $35.6 \%$ of females and $21.3 \%$ of males. When hair was washed on a daily basis, the infestation rate was $11.8 \%$, compared to $27.6 \%$ when the washing was done irregularly. Teaching the community about personal hygiene and the availability of anti- pediculosis drugs could lead to the reduction in the prevalence of pediculosiscapitis among children. ${ }^{3}$

\section{Objectives:}

1. To compare the effectiveness of two methods of management of pediculosiscapitis.

2. To find the effectiveness of each methods of management of pediculosiscapitis.

\section{Hypotheses:}

$\mathrm{H}_{1}$ : There will be a significant difference between median scores of two methods of management of pediculosiscapitis.

$\mathrm{H}_{2}$ : There will be a significant difference between median scores of pre and post pediculosis management of perlice cream rinse $1 \%$.

$\mathrm{H}_{3}$ : There will be a significant difference between median scores of pre and post pediculosis management of wet combing. 


\section{Materials and methods}

In this study schools were selected by random method, children for identifying the prevalence of pediculosis were by purposive and for the management by random sampling technique.

Data collection instrument was, Tool 1 Section A: Information related to hair,

Section B:Observation checklist to diagnose the degree of infestation and to check the effectiveness of the management.

The content validity of the tool was obtained by submitting the tool to 7 experts. The reliability of the tool was established by administering on 20 children with Pediculosiscapitis. The reliability of the tool was established by using Sphereman rank correlation for the observation checklist and the value obtained was 0.99 and the tool was considered reliable. Pilot study was conducted to assess the feasibility and practicability.

The main study was conducted in Aided Higher Primary School, Herebettu and ZillaPanchayath Higher Primary School, Athrady in 2010. The pediculosiscapitis infestation among children was identified by visual inspection. The children were selected for the management, were informed to ask their mothers or care takers to come to school on a fixed date to explain the treatment. On that day the selected children were checked for the degree of infestation by using observation checklist and management was explained to them. Follow up was done on next two days to their house who were selected for the study. The effectiveness of the management was checked with the observation checklist in school after 14 days in wet combing and after 7 days in case of perlice cream rinse $1 \%$ application.

The obtained data were analyzed based on the objectives and the hypotheses by using descriptive and inferential statistics and hypothesis was tested at 0.05 level of significance.

\section{Results}

Section 1: Frequency and percentage distribution of information related to hair.

Analysis of the data obtained to describe the information related to hair is shown in Table 1.

Table 1: Frequency percentage distribution of information related to hair.

\begin{tabular}{|c|c|c|c|}
\hline & & \multicolumn{2}{|c|}{$\mathrm{N}=135$} \\
\hline Sl. No & Information related to hair & f & $\%$ \\
\hline 1 & $\begin{array}{l}\text { Hair length (in centimetres) } \\
\leq 38 \\
>38\end{array}$ & $\begin{array}{l}54 \\
81\end{array}$ & $\begin{array}{l}40 \\
60\end{array}$ \\
\hline 2 & $\begin{array}{l}\text { Cosmetic used for the hair wash } \\
\text { Shampoo } \\
\text { Soap }\end{array}$ & $\begin{array}{c}14 \\
121\end{array}$ & $\begin{array}{l}10.4 \\
89.6\end{array}$ \\
\hline 3 & $\begin{array}{l}\text { Sharing of comb } \\
\text { No } \\
\text { Yes }\end{array}$ & $\begin{array}{c}6 \\
129\end{array}$ & $\begin{array}{c}4.4 \\
95.6\end{array}$ \\
\hline 4 & $\begin{array}{l}\text { Number of hair washes/ Week } \\
\leq 4 \\
>4\end{array}$ & $\begin{array}{c}109 \\
26\end{array}$ & $\begin{array}{l}80.7 \\
19.3\end{array}$ \\
\hline
\end{tabular}

Data presented in table 1 show that majority of the children had hair length more than $38 \mathrm{~cm}(60 \%)$, were using soap for the hair wash $(89.6 \%)$,were sharing the comb among the family members $(95.6 \%)$ and had less than 4 hair washes/ week(80.7\%).

Section 2: Frequency distribution of sample in terms of degree of infestation before and after the management

Data obtained to describe the degree of infestation before and after the treatment of pediculosiscapitis with perlice cream rinse $1 \%$ and wet combing are shown in Table $2 \& 3$.

Table 2: Frequency and percentage distribution of degree of infestation before and after the treatment in group I (perlice cream rinse 1\%)

\begin{tabular}{|c|c|c|c|c|}
\hline \multirow{2}{*}{ Degree of infestation } & & & & $=40$ \\
\hline & \multicolumn{2}{|c|}{ Before treatment } & \multicolumn{2}{|c|}{ After treatment } \\
\hline $1-<2$ Mild & 02 & 5.0 & 08 & 20.0 \\
\hline $2-<4$ Moderate & 06 & 15.0 & 04 & 10.0 \\
\hline $4-<6$ Severe & 31 & 77.5 & 00 & 00 \\
\hline
\end{tabular}


will be a significant difference between median scores of pre and post treatment of perlice cream rince $1 \%$.and wet combing.

\section{Discussion}

The present study findings revealed that majority $(60 \%)$ of the children had hair length more than $38(60 \%) \mathrm{cm}$, were using soap for the hair wash $(89.6 \%)$,were sharing the comb among the family members $(95.6 \%)$ and had less than 4 hair washes/ week $(80.7 \%)$. The present study findings supported by the study conducted in Maharashtra where the prevalence was higher $(36.27 \%)$ in children with poor personal hygiene as compared to those with satisfactory personal hygiene. The prevalence of head louse infestation was higher (41.97\%) in children with hair length more than $5 \mathrm{~cm}$ than in those whose hair length was less than $5 \mathrm{~cm}$ $(22.16 \%)$. This difference was found to be statistically highly significant $(\mathrm{p}<0.05)^{-1}$

The present study findings revealed that the perlice cream rinse $1 \%$ shown a better outcome than wet combing. $(Z=-2.775, P<0.05)$. The similar result reported with the use of other pediculicide in a pragmatic randomised controlled trial which was conducted tocompare the effectiveness of wet combing with Malathion for treatment of head lice in UK. Seventy four children completed the study and 72 were included in the analysis. The cure rate was 38\% (12 of 32) for bug-busting and 78\% (31 of 40) for Malathion. Children assigned bug-busting were $2.8(95 \%$ CI $1.5-5 \cdot 2)$ times more likely than those assigned malathion to have lice at the end of treatment $(\mathrm{p}=0.0006)$. The study concluded thatmalathion lotion was twice as effective as bug-busting. ${ }^{4}$

Similar finding reported by a study conducted to assess the prevalence of head louse infestation and the effectiveness of $1 \%$ permethrin against head lice in Kosovar refugees. Study concluded that $1 \%$ permethrin treatment was effective in louse control in this refugee population. ${ }^{5}$

The present study findings contradicted by the findings of a single blind randomised comparative study which revealed that the cure rate for malathion and permethrin were $17 \%$ and $10 \%$ respectively. The cure rate for the bug buster kit was significantly greater than that for the pediculicides( $57 \%$ versus 13\%; relative risk 4.4, 95\% confidence interval 2.3 to 8.5 ). Study concluded that the bug buster kit was the most effective over the counter treatment for head louse infestation in the community when compared with the pediculicides. ${ }^{6}$

\section{References}

[1]. SathyamoorthyTS,Sachdeva NL, Kabra SC, Ganguly SS. A prevalence study of pediculushumanuscapitis infestation among children in a slum area of Pune. Indian Journal of Community Medicine 1986;12(2):209-13.

[2]. Sokoff F. Identification and management of pediculosis.U S National Library and National Institute of Health.1994 Aug;19(8):62-4.

[3]. Bhatia V, Nayan S. Prevalence of pediculosiscapitis among children in a rural community. Indian Journal of Maternal and child health 1997 Apr;8(2):39-41.

[4]. Jan DM, Ineke B, Sara W, Robert VS, Filip M. Wet combing versus traditional scalp inspection to detect head lice in school children. BMJ 2000 Nov;321:1187-1188.

[5]. Manjrekar RR, Patridge SK, Korman AK, Barwick RS, Juranek DD. Efficacy of 1\% permethrin for the treatment of head louse infestations among Kosovar Refugees. Mil Med 2000;165:698-700

[6]. Hill N, Moor G, Cameron MM, Butlin A, Preston S, Williamson et al. Single blind, randomised,comparative study of the bug buster kit and over the counter pediculicide treatments against head lice in the United Kingdom. BMJ 2005 Aug;331:384-387. 